the advantages of ionic liquids and solid insulators, including manipulation at room temperature ${ }^{7}$. Lee et al. ${ }^{2}$ now demonstrate free-standing, rubber-like ion gels that can be cut with a razor blade and stuck onto several semiconductor materials to produce a transistor. Their approach therefore offers a neat way of making transistors.

The macromolecular components of ion gels are block copolymers - polymers in which two or more chemically distinct monomer units are linked together. In their experiments, Lee et al. made free-standing 0.6-millimetre-thick films of ion gels by using a weight ratio of block copolymer to ionic liquid of $1: 4$. The capacitance of the EDL that formed at the interface between the semiconductor and the ion gel was as high as $10 \mu \mathrm{F} \mathrm{cm}^{-2}$. Similar free-standing iongel films have been used as solid electrolytes in lithium-ion batteries. But Lee et al. used such films as gate insulators in transistors; gate insulators separate the transistor's semiconducting channel from its gate electrode (Fig. 1). What's more, the authors demonstrated transistors made from both typical organic and inorganic semiconductors; these showed current based on 'holes' (notional particles formed by the absence of electrons) and electrons, respectively. This method can thus be applied to any type of semiconducting material.
Because ion gels can be made in many forms (there are large numbers of ionic liquids and polymers), they could be used to obtain diverse novel device structures and functionalities ${ }^{7}$. For instance, printing of ion gels on substrates has already been demonstrated ${ }^{8}$. Finally, combinations of ion gels and a variety of electronic conductors - not only semiconductors but also metals, superconductors and magnets - will make 'iontronics', electronics based on ionic functions, a rich field of materials science.

Masashi Kawasaki and Yoshihiro Iwasa are at the Quantum Phase Electronics Research Center and in the Department of Applied Physics, University of Tokyo, Tokyo 113-8656, Japan, and at the RIKEN-Advanced Science Institute, Wako, Japan.

e-mail:kawasaki@ap.t.u-tokyo.ac.jp

1. Brattain, W. H. \& Gibney, R. B. US patent 2,524,034 (1948).

2. Lee, K. H. et al. Adv. Mater. 24, 4457-4462 (2012).

3. von Helmholtz, H. Pogg. Ann. LXXXIX, 211 (1853).

4. Nakano, M. et al. Nature 487, 459-462 (2012).

5. Ueno, K. et al. Nature Mater. 7, 855-858 (2008).

6. Yamada, Y. et al. Science 332, 1065-1067 (2011).

7. Lodge, T. P. Science 321, 50-51 (2008).

8. Cho, J. H. et al. Nature Mater. 7, 900-906 (2008).

9. Leger, J., Berggren, M. \& Carter, S. Iontronics: Ionic Carriers in Organic Electronic Materials and Devices (CRC, 2010).

\title{
When more is less
}

\section{A tightly regulated enzyme balances energy production and the synthesis of macromolecules from glucose in cancer cells. Upsetting this balance by stimulating the enzyme's activity can suppress tumour growth in mice.}

\section{LEI JIANG \& RALPH J. DEBERARDINIS}

$\mathrm{T}$ The development of cancer is associated with a suite of metabolic changes that support the energetic and biosynthetic requirements of tumour-cell proliferation. Many of these changes are stimulated by the same genetic mutations that drive tumorigenesis, suggesting that normalizing the tumour cells' metabolism by pharmacological means could suppress cancer progression. An enhancement of glycolysis, a cellular pathway that produces both energy and precursors of macromolecules, is a classic metabolic hallmark of cancer, making the glycolytic pathway an attractive target in which to test this hypothesis. Writing in Nature Chemical Biology, Anastasiou et al. ${ }^{1}$ show that targeting a form of the enzyme pyruvate kinase that is commonly found in tumour cells can redistribute the fate of glucose-derived metabolites away from biosynthetic pathways, and suppress tumour growth in mice.
The ability of the glycolytic pathway to produce ATP - the main cellular energytransfer molecule - is largely dependent on the activity of pyruvate kinases. There are various mammalian forms of pyruvate kinase, and tumour cells typically express the M2 isoform (PKM2), which shows reduced enzyme activity compared with other pyruvate kinases. This was initially thought to be somewhat paradoxical in cancer cells, in which abundant expression of glucose transporters and glycolytic enzymes conspires to enhance overall glycolysis. But one advantage of a bottleneck at the end of an otherwise active pathway is to force some of the intermediates it produces to accumulate, analogous to a partially closed valve at the bottom of a column of fluid (Fig. 1). It is now thought that the aggregation of metabolites behind this valve may promote their transfer into accessory pathways, including those that generate macromolecular building blocks and other substrates needed for cellular replication $^{2}$.

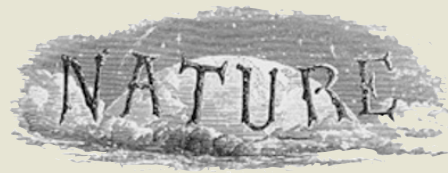

50 Years Ago

The third annual scientific meeting of the British Academy of Forensic Sciences ... was called to consider "The Hazards of the Road", a subject to which, having regard to the increasing public danger deriving from motor traffic, the sciences appear to have devoted disproportionately little research ... [P] sychological tests on those involved in accidents ... tended to show personality traits such as aggressiveness, carelessness, impulsiveness and the like which are not present to the same degree in those persons who have been free from accidents ... Older people tend to make up for their longer reaction times by driving more slowly ... Although as a man grows older, into middle age or further, his reaction time is slowed, his eyesight is not quite what it used to be and his hearing is impaired, he nevertheless becomes a better driver.

From Nature 29 September 1962

100 Years Ago

When nature was fashioning man the forces of natural selection made one hand more apt to perform skilled movements than the other. Why precisely it was the right hand that was chosen in the majority of mankind we do not know ... The fact that a certain proportion of mankind is left-handed, and that such a tendency is transmitted to only some of the descendants of a lefthanded person, might perhaps suggest that one half of mankind was originally left-handed and the other right-handed, and that the former condition was recessive in the Mendelian sense ... The superiority of one hand is as old as mankind, and is one of the factors incidental to the evolution of man.

From Nature 26 September 1912 
a Maximum cell proliferation

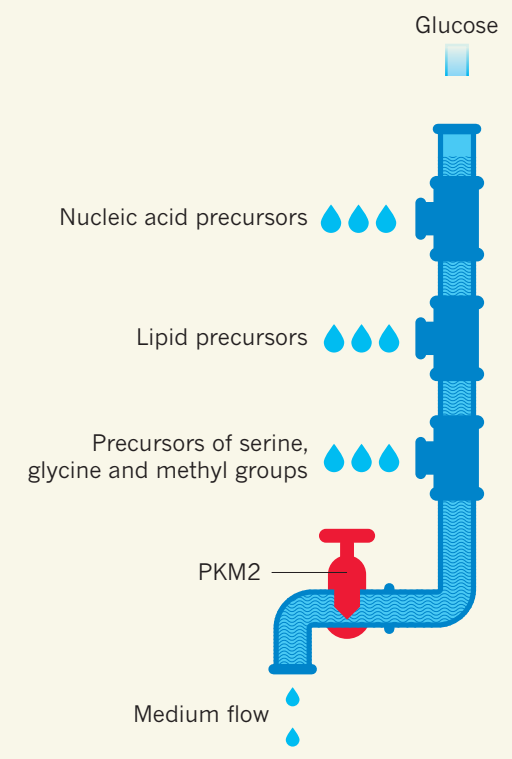

b Suppressed cell proliferation and tumour growth

Glucose

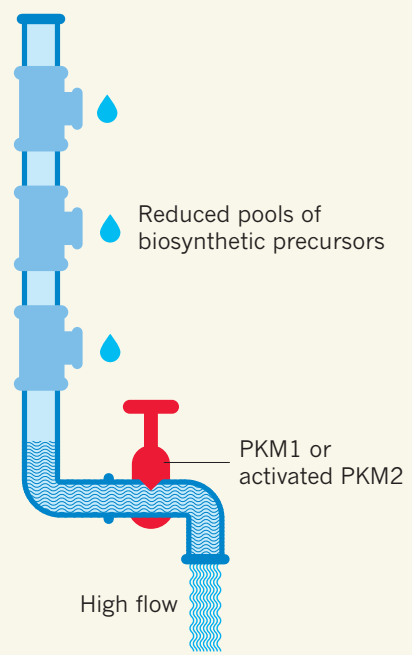

Figure 1 | A metabolic bottleneck. During glucose metabolism (glycolysis), the enzyme pyruvate kinase acts as a 'tap' that regulates the rate of flow out of the pathway. a, Cancer cells often express the M2 form of the enzyme (PKM2), which has lower enzymatic activity than other isoforms of pyruvate kinase. This, combined with the robust glycolysis that is also a hallmark of cancer cells, leads to an accumulation of glycolytic intermediates, including precursors of nucleic acids, lipids, serine, glycine and methyl groups. The accumulation in turn stimulates the biosynthetic pathways into which these intermediates feed, thereby contributing to the rapid cell proliferation seen in tumours. b, By contrast, enhancing pyruvate kinase activity by replacing PKM2 with the more active PKM1 isoform causes a decline in these pools of biosynthetic precursors and reduced cell proliferation ${ }^{3}$. Anastasiou et al. ${ }^{1}$ show that a similar tumoursuppressive effect can be achieved using small molecules that enhance PKM2 activity.

This explanation for the role of PKM2 in cancer gained momentum when it was demonstrated that replacing PKM2 in cancer cells with the more active PKM1 isoform resulted in higher overall activity of pyruvate kinase but reduced macromolecular synthesis and tumour growth in mice ${ }^{3}$. Further support came from studies showing that many of the processes that regulate PKM2 in cancer cells, such as binding of the enzyme by tyrosinephosphorylated proteins, act to reduce rather than stimulate its enzymatic activity ${ }^{4,5}$.

A possible implication of this picture of cancer-cell glycolysis is that stimulating PKM2 activity - in effect, opening the floodgates might impair tumour growth by reducing the availability of macromolecular precursors. Anastasiou et al. tested this idea by using small molecules that clamp the enzyme into a highly active tetramer configuration (a complex containing four subunits of the enzyme), and then analysing tumour-cell glycolysis. They found that cellular pools of glucose-dependent biosynthetic intermediates became depleted, and that flux through the pathways supplied by them was suppressed. Treatment with the PKM2 activator molecules also reduced the proliferation of cancer cells subjected to low oxygen levels, a condition that favours glycolysis. Finally, when the authors treated

tumour-bearing mice by oral administration of PKM2 activators, they found suppressed levels of biosynthetic intermediates in the tumours, and substantially slower tumour growth. Importantly, the small molecules also rendered PKM2 insensitive to blockade by tyrosine-phosphorylated proteins, suggesting that the agents might provide durable metabolic effects even in tumours that are programmed to reduce the activity of pyruvate kinases.

These studies of PKM2 activation provide a foundation for broader investigations of metabolism and cancer biology. Anastasiou and colleagues' results indicate that PKM2 affects biosynthesis in more subtle ways than those shown in Figure 1. For example, they found that activating PKM2 reduces the flux from glucose metabolism to fatty-acid synthesis, a pathway typically thought to involve the formation of pyruvate (a glycolytic intermediate molecule) by pyruvate kinases. Together, these new data imply that submaximal PKM2 activity helps to channel metabolites into biosynthetic pathways through mechanisms that do not simply involve an accumulation of intermediates, but that are not yet fully understood. It will be interesting to test whether modulating glycolysis through PKM2 activation or other mechanisms can be extended to suppress the proliferation of cells under conditions of normal oxygen levels, because such suppression would also be desirable in fast-growing areas of tumours that are well perfused by oxygen.

The push to understand PKM2 function, and now to manipulate it to suppress tumour growth, emphasizes a shift in focus in cancer metabolism research. Since Otto Warburg's early experiments on tumour glycolysis in the 1920 s, work in this field has largely centred on how tumours produce energy. But recent studies have taken a much broader view of the metabolic network in cancer cells, leading to a greater appreciation of the complexities of biosynthesis, reduction-oxidation homeostasis and other facets of metabolism that support cell survival and proliferation. These investigations have uncovered many unexpected roles for metabolism in cell signalling and the regulation of gene expression, extending the reach of metabolic enzymes into essentially every area of cell biology ${ }^{6}$. These newly identified functions are highly relevant to cancer. Consider, for example, that PKM2 also acts as an activator of gene expression, such that it has proliferation-promoting activities that are independent of its enzymatic function ${ }^{7}$.

Finally, Anastasiou et al. have illustrated an important concept in the metabolic control of tumorigenesis. They show that activating PKM2 suppresses cell proliferation - but severely inhibiting this enzyme is known to have similar effects, and can induce tumour regression in mice ${ }^{8,9}$. It is likely that the highest rates of cell proliferation are the result of a metabolic network in which a set of enzymes that, like PKM2, contribute to biosynthesis are precisely controlled, but whose maximal activation is counterproductive. These nodes may be the most easily disrupted control points in the metabolic network, and identifying them should offer the best opportunities for metabolic therapy in cancer.

Lei Jiang and Ralph J. DeBerardinis are at the Children's Medical Center Research Institute, University of Texas Southwestern Medical Center, Dallas, Texas 75390-8502, USA.

e-mail:ralph.deberardinis@utsouthwestern.edu

1. Anastasiou, D. et al. Nature Chem. Biol. 8, 839-847 (2012).

2. Mazurek, S., Boschek, C. B. \& Eigenbrodt, E. J. Bioenerg. Biomembr. 29, 315-330 (1997).

3. Christofk, H. R. et al. Nature $452,230-233$ (2008).

4. Christofk, H. R., Vander Heiden, M. G., Wu, N., Asara, J. M. \& Cantley, L. C. Nature 452, 181-186 (2008).

5. Hitosugi, T. et al. Sci. Signal. 2, ra73 (2009).

6. Lu, C. \& Thompson, C. B. Cell Metab. 16, 9-17 (2012).

7. Luo, W. et al. Cell 145, 732-744 (2011).

8. Vander Heiden, M. G. et al. Biochem. Pharmacol. 79, 1118-1124 (2010).

9. Goldberg, M. S. \& Sharp, P. A. J. Exp. Med. 209, 217-224 (2012).

The authors declare competing financial interests. See go.nature.com/e69jt8 for details. 